University of Nebraska - Lincoln

DigitalCommons@University of Nebraska - Lincoln

Stephen Ducharme Publications

Research Papers in Physics and Astronomy

April 1991

\title{
Observation of the photorefractive effect in a polymer
}

\author{
Stephen Ducharme \\ University of Nebraska - Lincoln, sducharme1@unl.edu \\ J.C. Scott \\ IBM Research Division, IBM Almaden Research Center, San Jose, California \\ R.J. Twieg \\ IBM Research Division, IBM Almaden Research Center, San Jose, California \\ W.E. Moerner \\ IBM Research Division, IBM Almaden Research Center, San Jose, California
}

Follow this and additional works at: https://digitalcommons.unl.edu/physicsducharme

Part of the Physics Commons

Ducharme, Stephen; Scott, J.C.; Twieg, R.J.; and Moerner, W.E., "Observation of the photorefractive effect in a polymer" (1991). Stephen Ducharme Publications. 21.

https://digitalcommons.unl.edu/physicsducharme/21

This Article is brought to you for free and open access by the Research Papers in Physics and Astronomy at DigitalCommons@University of Nebraska - Lincoln. It has been accepted for inclusion in Stephen Ducharme Publications by an authorized administrator of DigitalCommons@University of Nebraska - Lincoln. 


\title{
Observation of the Photorefractive Effect in a Polymer
}

\author{
Stephen Ducharme, J. C. Scott, R. J. Twieg, and W. E. Moerner \\ IBM Research Division, IBM Almaden Research Center, San Jose, California 95120-6099
}

(Received 13 November 1990)

\begin{abstract}
We report the first observation of the photorefractive effect in a polymeric material, the electro-optic polymer bisphenol- $A$-diglycidylether 4-nitro-1,2-phenylenediamine made photoconductive by doping with the hole-transport agent diethylamino-benzaldehyde diphenylhydrazone. The gratings formed exhibit dynamic writing and erasure, strong electric-field dependence, polarization anisotropy, and estimated space-charge fields up to $26 \mathrm{kV} / \mathrm{cm}$ at an applied field of $126 \mathrm{kV} / \mathrm{cm}$. Application of similar concepts should provide a broad new class of easily fabricated photorefractive materials.
\end{abstract}

PACS numbers: $42.65 . \mathrm{Hw}, 72.20 . \mathrm{Jv}, 78.20 . \mathrm{Jq}, 78.65 . \mathrm{Hc}$

Twenty years have elapsed since the discovery of the photorefractive (PR) effect and the early realization of its potential utility; ${ }^{1}$ for example, ${ }^{2}$ high-density opticaldata storage, many image-processing techniques, phase conjugation, simulations of neural networks and associative memories, and programmable interconnection. The commercial introduction of such technology has been hindered by the difficulty of preparing and processing the inorganic crystals (such as $\mathrm{BaTiO}_{3}$ and $\mathrm{Sr}_{x} \mathrm{Ba}_{1-x} \mathrm{Nb}_{2} \mathrm{O}_{6}$ to name two examples) which show the effect. The purpose of this Letter is to describe the physical basis for, and properties of, the first of an entirely new class of easily fabricated PR materials, doped nonlinear optical polymers.

The PR effect requires the redistribution and trapping of charge in the bulk of a photoconducting insulator or semiconductor under nonuniform illumination. The electric fields associated with the trapped charge alter the index of refraction of the material through the electrooptic (EO) effect, thus producing a phase replica, or hologram, of the optical intensity distribution. The ingredients necessary for producing a PR phase hologram are, therefore, photoionizable charge generator, transporting medium, trapping sites, and dependence of the index of refraction upon space-charge field.

Recently, the PR effect was observed in a carefully grown doped organic crystal. ${ }^{3}$ In fact, in spite of possibly lower mobilities, organics in general have potential advantages over inorganic crystals since a reasonably large electro-optic response can be attained with far lower dielectric constant. ${ }^{4}$ The growth of doped organic crystals, however, is a very difficult process because most dopants are expelled during the crystal preparation. Polymers, on the other hand, can frequently be doped by simply mixing the various functional components; furthermore, they are ideally suited for future thin-film waveguide applications and can have nonlinear coefficients larger than $\mathrm{LiNbO}_{3}$, for example. ${ }^{5}$ We have made a polymeric PR material, the first to our knowledge, in order to illustrate the generality of this approach for the formulation of new photorefractive ma- terials. Polymers have been separately made electrooptic by poling guest or attached nonlinear chromophores 6,7 and made photoconducting at virtually any wavelength by doping with charge-generation and -transporting agents. $^{8,9}$

In this work, the EO material is the partially crosslinked epoxy polymer bisA-NPDA composed of bisphenol- $A$-diglycidylether (bisA) reacted with the nonlinear chromophore 4-nitro-1,2-phenylenediamine (NPDA) which has been studied previously in its crosslinked form as a stable second-order material. ${ }^{10}$ The polymer had a glass transition temperature $T_{g}=65^{\circ} \mathrm{C}$ and number and weight average molecular weights $\bar{M}_{n}$ $=2200$ and $\bar{M}_{w}=6900$, respectively. The bisA-NPDA polymer acquires second-order nonlinearity when the NPDA chromophores are oriented in an electric field. It will be shown below that the NPDA also provides optical absorption for the charge-generation process.

Monopolar charge transport is facilitated by addition of the hole-transport agent diethylamino-benzaldehyde diphenylhydrazone (DEH) to the polymer to form connected pathways for the hopping motion of holes as is done in the charge-transport layer of electrophotographic photoconductors. ${ }^{9}$ In molecularly doped polymers, trapping centers are invariably present due to defects and conformational disorder inherent in the amorphous structure. Therefore, for these initial studies, no additional traps were added. The polymer and DEH were codissolved in the appropriate ratio in PM (propylene glycol monomethyl ether) or PM acetate, stirred thoroughly, and filtered. Samples were made by dripping 1-3 $\mathrm{ml}$ of the polymer-DEH-solvent mixture onto two indium-tin-oxide-coated glass plates set on a hot plate, drying at $95^{\circ} \mathrm{C}$ for $30 \mathrm{~min}$ to remove most of the solvent, and then pressing the two plates together face to face. Fifteen samples $178-533 \mu \mathrm{m}$ thick prepared in the same manner exhibited similar EO, photocurrent, and PR properties.

The separate contributions that the host polymer and the hole-transport agent make to the photoconductivity are illustrated in Fig. 1. The absorbance of the sample 


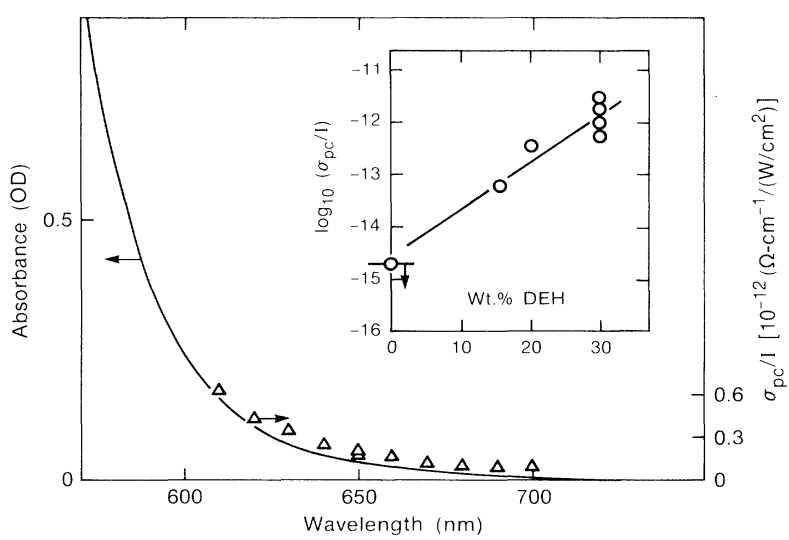

FIG. 1. Absorbance (left ordinate) and photosensitivity (right ordinate) of the bisA-NPDA polymer mixed with 30 wt \% DEH. Inset: The photosensitivity (at $647.1 \mathrm{~nm}, 1.4$ $\mathrm{W} / \mathrm{cm}^{2}$ illumination, $28 \mathrm{kV} / \mathrm{cm}$ field) as a function of $\mathrm{DEH}$ concentration. (The point at $0 \mathrm{wt} \%$ is an upper limit.)

in the red (left scale) is not significantly different from the undoped polymer since DEH absorbs in the blue. The strong absorption onset is due to the first electronic transition of the NPDA. The inset shows that the photosensitivity $\sigma_{\mathrm{pc}} / I$ (photoconductivity per unit intensity) increases rapidly once sufficient DEH is added, consistent with the establishment of a conductive network for charge transport. The measured photocurrents were approximately linear in the bias voltage and in intensity and were determined to be free from significant contributions due to heating. The photosensitivity of a sample made with $30 \mathrm{wt} \%$ DEH has a spectral dependence which closely follows the measured optical absorbance of the sample (Fig. 1, right scale), suggesting that optical excitation of the NPDA chromophores is the first step in charge generation.

Usually, epoxy polymers are aligned by poling and then cross-linked at elevated temperatures to produce stable nonlinearity. ${ }^{10}$ Attempts to cure our samples produced a loss of optical quality perhaps due to phase separation. (DEH segregation may also account for a slow loss of photoconductivity of $\simeq 50 \%$ per week at $20^{\circ} \mathrm{C}$ and $50 \%$ per 2 months at $7^{\circ} \mathrm{C}$.) Hence, we chose not to cross-link the samples, but to perform most experiments at room temperature with a dc poling electric field present both to permit external control of the EO response and to simplify sample preparation.

We measure the EO coefficients of the samples at low frequencies $(10-10000 \mathrm{~Hz})$ using a Mach-Zehnder interferometer. ${ }^{11}$ The observed response was proportional to the applied field $E_{0}$ except for a small amount of hysteresis at fields below $5 \mathrm{kV} / \mathrm{cm}$. Upon changing or reversing the applied field, the observed EO response rises in a matter of minutes to approximately $90 \%$ of its longtime (i.e., many hours) value as the nonlinear chromophores are aligned. The slow time dependence of the ob-

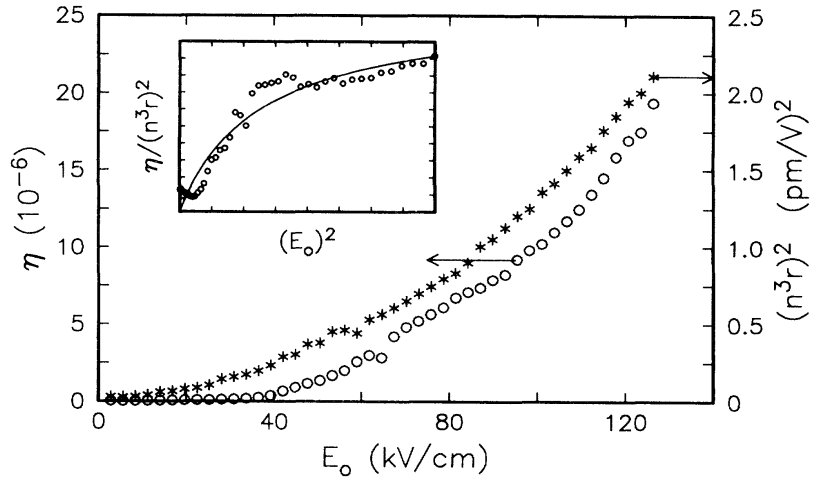

FIG. 2. Right ordinate: Square of the electro-optic response ( $n^{3} r_{\text {eff, with }} n$ the index of refraction) for $p$-polarized $632.8-\mathrm{nm}$ radiation incident at an external angle of $60^{\circ}$ on a $356-\mu \mathrm{m}$ thick film. Left ordinate: Diffraction efficiency at $647.1 \mathrm{~nm}$ for the same sample as a function of applied field. Inset: The ratio of the latter to the former, which is proportional to $E_{\mathrm{sc}}^{2}$.

served EO response confirms that the source of the nonlinearity is molecular alignment rather than a Kerr effect. The right ordinate of Fig. 2 shows the steadystate EO response of a 30-wt\% DEH mixture. The effective EO coefficient (neglecting birefringence) $r_{\text {eff }}$ $=r_{33} \sin ^{2} \alpha+r_{13} \cos ^{2} \alpha$ is a linear combination of the longitudinal $r_{33}$ and transverse $r_{13}=r_{23}$ coefficients where the 3 direction is the direction of the applied electric field (normal to the film plane) and $\alpha=32.1^{\circ}$ (for $n=1.63$ ) is the angle of propagation within the film.

Thus, three of four necessary ingredients for a PR material (absorption, transport, and EO response) can be easily measured in our polymer mixtures. The polymer would be expected to supply a large density of traps, which may be polymer chain ends of other defects, but there is no guarantee that these traps will be photoionizable as required for the production of an erasable PR space-charge field. ${ }^{2}$ The presence of the PR effect and therefore a sufficient number of useful traps has been verified in the polymer films by volume holography. Two mutually coherent Gaussian "writing" beams at $\lambda$ $=647.1 \mathrm{~nm}$ with equal intensities of $13 \mathrm{~W} / \mathrm{cm}^{2}$ were intersected in the sample at incidence angles of $30^{\circ}$ and $60^{\circ}$ (in air), respectively, thus producing interference fringes with spacing $\Lambda_{g}=1.6 \mu \mathrm{m}$ oriented $25^{\circ}$ from the film plane. ${ }^{12}$ A third, much weaker, "reading" beam directed opposite to the $60^{\circ}$ writing beam was Bragg diffracted from the grating formed by the writing beams. The diffraction efficiency $\eta$ was recorded as the ratio of diffracted to incident reading beam power. The writing beams could be either polarization, but $p$-polarized readout produced $\simeq 6 \pm 2$ times stronger signals, consistent with the expected anisotropy of the electro-optic tensor.

The influence of $E_{0}$ on $\eta$ is shown in Fig. 2, left scale, where at each point the molecular alignment was allowed 
to reach steady state in the field for $10 \mathrm{~min}$. The value of $\eta=\left(n^{3} r_{\mathrm{eff}} \pi L G E_{\mathrm{sc}} / 2 \lambda\right)^{2}$ increases rapidly with $E_{0}$ through increases in both the PR space-charge field $E_{\mathrm{sc}}$ and the EO response $n^{3} r_{\text {eff }}$, where $L=356 \mu \mathrm{m}$ and $G$ is a polarization and geometrical factor equal to 0.86 . The PR space-charge field in the standard model ${ }^{2,13,14}$ is

$$
E_{\mathrm{sc}}^{2}=\frac{E_{d}^{2}+E_{0 g}^{2}}{(1+A)^{2}+A^{2}\left(E_{0 g} / E_{d}\right)^{2}},
$$

where $E_{0 g}$ is the component of $E_{0}$ along the grating wave vector, $E_{d}=k_{g} k_{B} T / e=1.0 \mathrm{kV} / \mathrm{cm}$, and $k_{g}=2 \pi / \Lambda_{g}$ $=3.9 \times 10^{4} \mathrm{~cm}^{-1}$. The inset to Fig. 2 shows $\eta$ divided by $\left(n^{3} r_{\text {eff }}\right)^{2}$, which should be proportional to $E_{\text {sc. }}^{2}$. The solid line is a least-squares fit by Eq. (1) with two adjustable parameters: an overall multiplicative factor ${ }^{15}$ and $A$ $=0.034$ which yields the effective density of photorefractive traps $N_{\mathrm{pr}}=k_{g}^{2} \varepsilon \varepsilon_{0} k_{B} T / A e^{2}=1.9 \times 10^{15} \mathrm{~cm}^{-3} \quad(\varepsilon$ $=2.9$ ). The fit provides a good description of the shape of the experimental data. The maximum $E_{\mathrm{sc}}$ reached, at an applied field of $126 \mathrm{kV} / \mathrm{cm}$, from Eq. (1) is $26 \mathrm{kV} / \mathrm{cm}$, a value which is larger than is generally attainable in inorganic PR materials. Clearly, the low dielectric constant of the polymer allows a larger field for the small trapped charge density $N_{\mathrm{pr}}$ than might be possible in inorganics. The low values of $\eta$, on the other hand, are to be expected due to the short optical path lengths and relatively small EO coefficients of this particular polymer. In preliminary measurements with the more highly nonlinear polymer NNDN-NAN (see Ref. 16 for structure and nonlinear properties) also doped with $\mathrm{DEH}, \eta$ values up to $10^{-3}$ have been observed in $350-\mu \mathrm{m}$-thick samples.

To further establish that the observed gratings were indeed electro-optic in origin, we recorded gratings at large applied voltage, removed the writing beams, and observed the diffracted signal as the applied voltage was altered, with typical results shown in Fig. 3. From $a$ to $b$, the grating was recorded for $2.5 \mathrm{~min}$ in a field of $-84.3 \mathrm{kV} / \mathrm{cm}$ which had been applied long in the past. The fluctuations in the signal as it rises to its steady-state value are due to slow changes in the optical paths of the writing beams. At $b$, both writing beams and the applied field were switched off resulting in a rapid decrease in the signal. We attribute this to initial relaxation of the chromophore alignment, without loss of the space charge (except for the slow erasure by the much weaker reading beam), because the signal returns upon application of a field of either sign ( $c$ and $d$ in Fig. 3). The dynamics for signal recovery are consistent with that required for reorientation of the nonlinear chromophores and are far slower than the charging time of the circuit, thus ruling out strong Kerr effect contributions. At $e$, one of the writing beams was turned back on to erase the spacecharge field in a time of order $30 \mathrm{~s}$, which should be compared with the dark lifetime of order $500 \mathrm{~s}$, limited by the dark conductivity of the material. Thus, Fig. 3

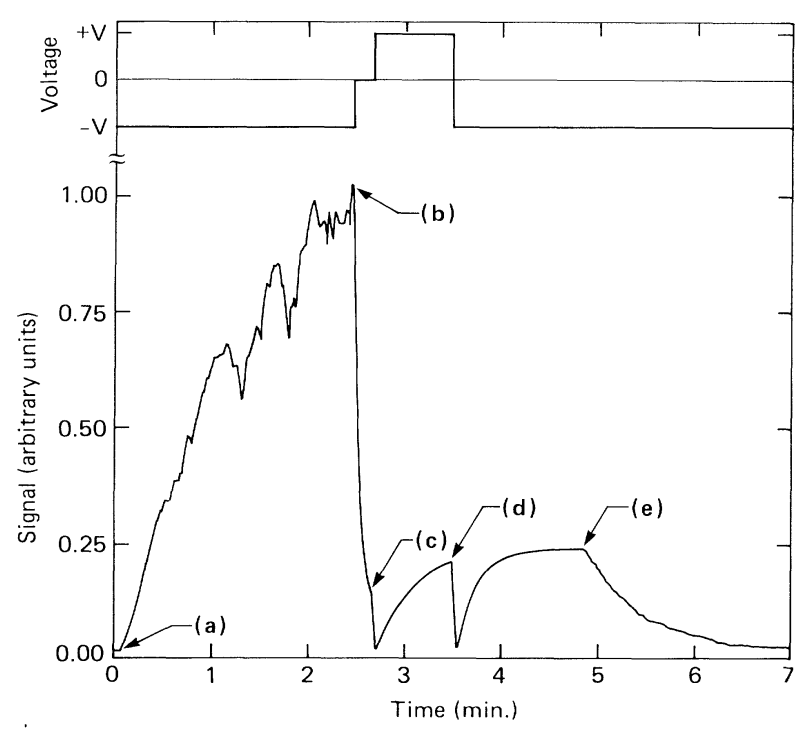

FIG. 3. Effect of applied field on grating readout by a weak reading beam: $a-b$, writing the grating in the presence of a (previously applied) negative voltage; $b-c$, writing beams and applied voltage turned off; $c-d$, positive voltage applied; $d-e$, negative voltage applied; and $e$ on, grating erasure by one strong beam.

establishes the relative independence of space-charge formation and erasure from the molecular alignment whose only purpose is to permit readout, and reinforces the interpretation in terms of a photorefractive mechanism.

The holographic gratings could be repeatedly written and erased dozens of times without noticeable degradation. The need for large external electric fields during grating recording can be understood because an applied field is necessary to assist in separation of the photogenerated charges. In the absence of an applied field during writing, Eq. (1) shows that $E_{\mathrm{sc}}$ is equal to $E_{d}=1.0$ $\mathrm{kV} / \mathrm{cm}$ which would yield $\eta \simeq 10^{-8}$ even with a readout field of $126 \mathrm{kV} / \mathrm{cm}$. As an additional test, we attempted to record gratings with $E_{0}=0$ and, after blocking the writing beams so that any resulting space charge would remain fixed, applied a large $E_{0}$. No signal was observed larger than the approximately $(1-5) \times 10^{-8}$ background level. This is also consistent with the well-known pronounced dependence of the photogeneration rate ${ }^{17}$ and the mobility ${ }^{18}$ on the external field in organic photoconductors.

The PR effect has a number of unique features which distinguish it from any other known mechanism of grating formation. The clearest single signature of PR gratings, dynamic two-beam energy coupling, ${ }^{19}$ has not yet been observed in our polymers because of the low diffraction efficiencies achieved. However, the electricfield dependence of the grating formation and readout, the correlation with photoconductivity and EO response, and the cyclic erasability eliminate the possibility that 
the observed effect is due to any mechanism other than photorefraction, such as photochromism, photochemical effects, or photophysical changes in molecular structure. Hence, the observations detailed here, taken together, provide unequivocal evidence that the molecularly doped polymer system studied is indeed photorefractive.

The ease of formulation of this material ensures that future efforts by synthetic chemists and optical physicsts alike will provide many new examples of photorefractive polymers. From such interdisciplinary studies, the detailed mechanistics, ultimate limitations, and possibilities of this new class of materials can be deduced.

We thank B. Reck for polymer synthesis, D. Jungbauer for solvent and poling advice, and G. C. Bjorklund and D. M. Burland for critical support and encouragement. This work was supported in part by the U.S. Office of Naval Research.

${ }^{1}$ F. S. Chen, J. Appl. Phys. 38, 3418 (1967).

${ }^{2}$ Photorefractive Materials and Their Applications, edited by P. Günter and J.-P. Huignard (Springer-Verlag, Berlin, 1988), Vol. 1; Photorefractive Materials and Their Applications, edited by P. Günter and J.-P. Huignard (SpringerVerlag, Berlin, 1989), Vol. 2.

${ }^{3}$ K. Sutter, J. Hulliger, and P. Günter, Solid State Commun. 74, 867 (1990).

${ }^{4}$ S. Ducharme, J. C. Scott, R. J. Twieg, and W. E. Moerner, postdeadline paper to the Optical Society of America Annual Meeting, Boston, Massachusetts, 5-9 November 1990 (unpublished).

${ }^{5}$ K. D. Singer, M. G. Kuzyk, W. R. Holland, J. E. Sohn, S. J. Lalama, R. B. Comizzoli, H. E. Katz, and M. L. Schilling, Appl. Phys. Lett. 53, 1800 (1988).

${ }^{6}$ D. J. Williams, in Nonlinear Optical Properties of Organic Molecules and Crystals I, edited by D. S. Chemla and J. Zyss
(Academic, Orlando, 1987), pp. 405-435.

${ }^{7}$ G. R. Möhlmann, in Organic Materials for Non-linear Optics, edited by R. A. Hann and D. Bloor (Royal Society of Chemistry, London, 1989), p. 275.

${ }^{8}$ M. Lardon, E. Lell-Döller, and J. W. Weigl, Mol. Cryst. 2, 241 (1967).

${ }^{9}$ L. B. Schein, A. Rosenberg, and S. L. Rice, J. Appl. Phys. 60, 4287 (1986).

${ }^{10}$ M. Eich, B. Reck, D. Y. Yoon, C. G. Willson, and G. C. Bjorklund, J. Appl. Phys. 66, 3241 (1989).

${ }^{1 ' S}$. Ducharme, J. Feinberg, and R. Neurgaonkar, IEEE J. Quantum Electron. 23, 2116 (1987).

${ }^{12}$ This geometry was chosen to enhance the sensitivity by providing a reasonable projection of the space-charge field along the film normal, i.e., the poling direction.

${ }^{13}$ J. Feinberg, D. Heiman, A. R. Tanguay, Jr., and R. W. Hellwarth, J. Appl. Phys. 51, 1297 (1980); 52, 537 (1980).

${ }^{14}$ A. Twarowski, J. Appl. Phys. 65, 2833 (1989), derived the $E_{\mathrm{sc}}$ expected in the case of organic photoconductors with Onsager geminate recombination. Though the functional form of $E_{\text {sc }}$ differs from Eq. (1), the data in Fig. 2 are not sufficiently accurate to distinguish the models.

${ }^{15}$ Though the data of Fig. 2 were measured on the same sample, considerable scatter in EO coefficients $(25 \%)$ was observed due to sample inhomogeneity. Also, the EO coefficient of the polymer was approximately $10 \%$ higher at 632.8 than at 647.1 $\mathrm{nm}$ and separate measurement revealed that the piezoelectric coefficient $d_{33} \sim+0.2 \mathrm{pm} / \mathrm{V}$, which may contain a contribution from electrode attraction, also made a significant (10\%-30\%) contribution to the observed EO measurements. The data in Fig. 2 are not corrected for these observations, thus an overall fitting factor of $\approx 2.5$ is not unreasonable.

${ }^{16}$ D. Jungbauer, B. Reck, R. Twieg, D. Y. Yoon, C. G. Wilson, and J. D. Swalen, Appl. Phys. Lett. 56, 2610 (1990).

${ }^{17}$ T. E. Goliber and J. H. Perlstein, J. Chem. Phys. 80, 4162 (1984).

${ }_{18}$ J. X. Mack, L. B. Schein, and A. Peled, Phys. Rev. B 39, 7500 (1989).

${ }^{19}$ V. L. Vinetskii, N. V. Kukhtarev, S. G. Odulov, and M. S. Soskin, Usp. Fiz. Nauk 129, 113 (1979) [Sov. Phys. Usp. 22, 742 (1979)]. 